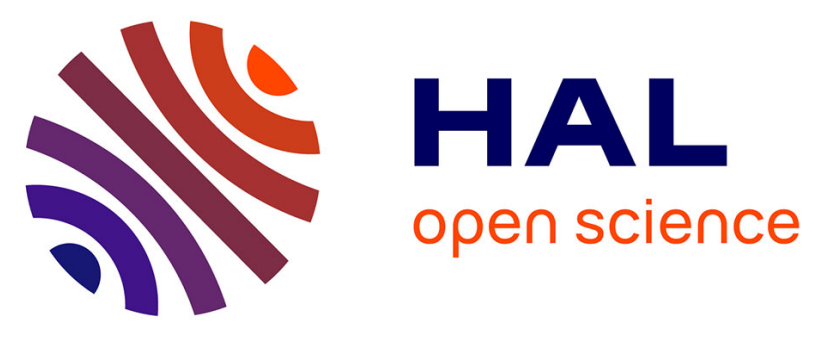

\title{
Lattice strain in irradiated materials unveils a prevalent defect evolution mechanism
}

Aurélien Debelle, Jean-Paul Crocombette, Alexandre Boulle, Alain Chartier,

Thomas Jourdan, Stéphanie Pellegrino, Diana Bachiller-Perea, Denise

Carpentier, Jayanth Channagiri, Tien-Hien Nguyen, et al.

\section{To cite this version:}

Aurélien Debelle, Jean-Paul Crocombette, Alexandre Boulle, Alain Chartier, Thomas Jourdan, et al.. Lattice strain in irradiated materials unveils a prevalent defect evolution mechanism. Physical Review Materials, 2018, 2 (1), pp.013604. 10.1103/PhysRevMaterials.2.013604 • hal-02193081

\section{HAL Id: hal-02193081 \\ https://hal.science/hal-02193081}

Submitted on 24 Jul 2019

HAL is a multi-disciplinary open access archive for the deposit and dissemination of scientific research documents, whether they are published or not. The documents may come from teaching and research institutions in France or abroad, or from public or private research centers.
L'archive ouverte pluridisciplinaire HAL, est destinée au dépôt et à la diffusion de documents scientifiques de niveau recherche, publiés ou non, émanant des établissements d'enseignement et de recherche français ou étrangers, des laboratoires publics ou privés. 


\section{Lattice strain in irradiated materials unveils a prevalent defect evolution mechanism}

Aurélien Debelle $^{1,2 *}$, Jean-Paul Crocombette ${ }^{3}$, Alexandre Boulle ${ }^{4}$, Alain Chartier ${ }^{5}$, Thomas Jourdan ${ }^{3}$, Stéphanie Pellegrino ${ }^{2}$, Diana Bachiller-Perea ${ }^{1}$, Denise Carpentier ${ }^{3}$, Jayanth Channagiri ${ }^{4}$, Tien-Hien Nguyen ${ }^{1}$, Frédérico Garrido ${ }^{1}$, Lionel Thomé ${ }^{1}$

1. Centre de Sciences Nucléaires et de Sciences de la Matière, Université Paris-Sud, CNRS/IN2P3, Université Paris-Saclay, 91405 Orsay, France.

2. CEA, DEN, Service de Recherches de Métallurgie Physique, Laboratoire JANNUS, Université ParisSaclay, 91191 Gif-sur-Yvette, France.

3. CEA, DEN, Service de Recherches de Métallurgie Physique, Université Paris-Saclay, 91191 Gif-surYvette, France.

4. Science des Procédés Céramiques et Traitements de Surface, CNRS UMR 7315, Centre Européen de la Céramique, 12 rue Atlantis, 87068 Limoges, France.

5. CEA, DEN, DPC, SCCME, Université Paris-Saclay, 91191 Gif-Sur-Yvette, France. 


\section{Abstract}

Modification of materials using ion beams has become a widespread route to improve or design materials for advanced applications, from ion doping for microelectronic devices to emulation of nuclear reactor environments. Yet, despite decades of studies, major issues regarding ion/solid interactions are not solved, one of them being the lattice-strain development process in irradiated crystals. In this work, we address this question using a consistent approach that combines X-ray diffraction (XRD) measurements with both molecular dynamics (MD) and rate equation cluster dynamics (RECD) simulations. We investigate four distinct materials that differ notably in terms of crystalline structure and nature of the atomic bonding. We demonstrate that these materials exhibit a common behaviour with respect to the strain development process. In fact, a strain build-up followed by a strain relaxation is observed in the four investigated cases. The strain variation is unambiguously ascribed to a change in the defect configuration, as revealed by MD simulations. Strain development is due to the clustering of interstitial defects into dislocation loops, while the strain release is associated with the disappearance of these loops through their integration into a network of dislocation lines. RECD calculations of strain depth profiles, which are in agreement with experimental data, indicate that the driving force for the change in the defect nature is the defect clustering process. This study paves the way for quantitative predictions of the microstructure changes in irradiated materials.

Keywords: irradiation; strain; atomic scale; defects; X-ray diffraction; computation 


\section{Introduction}

The use of ion beams over a broad range of energies is well established as being a powerful tool to synthesize ${ }^{1,2}$, shape ${ }^{2-5}$, test ${ }^{6,7,8}$ and references therein and modify the properties ${ }^{1,9,10}$ of materials. Research and applications of ion beams encompass advanced electro-optical devices ${ }^{9,} 11,12$, engineered nanostructures $\mathrm{s}^{2,3,13}$, strain engineering ${ }^{14-16}$, nuclear materials $\mathrm{s}^{17-19}$ and space exploration ${ }^{20,}$

${ }^{21}$. In most cases, the desired goals are enabled by the energy transfer from the energetic particles to atomic nuclei and/or electrons of the target ${ }^{22}$. Usually, deposited energy leads to defect generation and damage accumulation. These phenomena are sometimes desired, e.g., when the material resistance is tested or to improve the materials physical properties which includes mechanical ${ }^{1}$ and optical ${ }^{9}$ properties; they may also be a drawback, for instance in ion doping processes. In any case, understanding the mechanisms of damage formation and accumulation in materials as a result of energy deposition clearly appears as a crucial task to tackle.

Damage accumulation in irradiated materials is commonly studied through the analysis of phenomenological parameters such as the damage fraction in Rutherford backscattering spectrometry in channelling mode, the intensity variation of Raman spectroscopy lines or the Doppler broadening of positron-electron annihilation peaks ${ }^{8}$. These parameters convey information regarding random lattice displacements in the materials but establishing a connection with the actual defect structure and density is not straightforward, neither is the quantitative comparison with computational modelling. Besides random lattice displacements, defects also produce correlated displacements, i.e. lattice elastic strain, the analysis of which can provide essential information regarding the nature and the concentration of the defects ${ }^{23-26}$. Moreover, direct and quantitative comparisons can be made with the information derived from computational data ${ }^{26,27}$. X-ray diffraction (XRD) is highly sensitive to the atomic displacement field within the crystal, and hence is a dedicated tool to measure the strain consecutive to ion irradiation. 


\section{Current state of knowledge and issues addressed in this work}

Since the early 1980 's, research works dealing with the study, by XRD, of the strain induced by ion (or even neutron and electron) energy deposition have covered a wide range of materials, including mainly semiconductors ${ }^{24,28-32}$ and insulators ${ }^{16,33-36}$ but also, though more recently, metals ${ }^{15,}$ ${ }^{37-39}$. In all these works, two steps in the development of strain are observed. In the first step, particle irradiation leads to elastic strain build-up. The nature of the second step depends on the material. On the one hand, some materials (such as pyrochlores) undergo a crystalline-to-crystalline phase change ${ }^{32,40,41}$; in most semiconductors, long-range atomic order is progressively lost, eventually leading to a complete amorphization ${ }^{24,28}$. In both cases, strain measurement is no more possible in the initial structure which has disappeared. It is yet worth mentioning that the study of the strain evolution in irradiated silicon allowed to point out radiation-enhanced plastic flow in this material ${ }^{42}$. On the other hand, in materials that retain their original crystalline structure, the microstructure is nonetheless also severely affected, and two situations, that at first might seem conflicting, are observed as far as the strain evolution is concerned. In the first situation, a strain saturation phenomenon is noticed at high ion fluence (number of ions per unit surface) ${ }^{30-32}$, and it is ascribed to a balance between the strain induced by interstitial and vacancy defects ${ }^{30}$ or to the trapping of point defects at stacking faults ${ }^{31}$. However, it is worth mentioning that in most cases, the difficulty of analysing the complex XRD data at high ion fluence is likely one of the reasons why a saturation was assumed. In the second situation, which is the most widely observed ${ }^{15,25,33-36,38,39}$, a strain relaxation is measured. The most common explanation for this strain relief is the formation of extended defects. Note that when irradiated at intermediate temperatures, amorphization is delayed or even prevented in semiconductors like $\mathrm{Si}$ or $\mathrm{SiC}$, and strain relaxation also takes place by dislocation formation $^{43-45}$. But this conclusion on the origin of the strain relaxation is based on additional

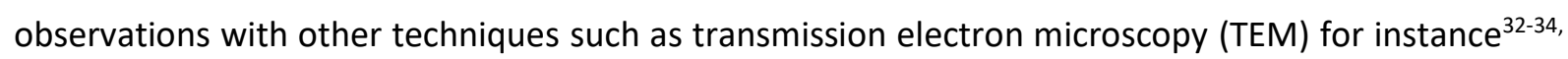
36, 38. Besides, owing to the different sensitivity of experimental techniques (e.g. XRD vs. TEM) on the 
nature, size and concentration of defects, demonstrating an indubitable link between a given defect structure and the measured strain level is a real challenge.

Strikingly, although crucial for the understanding of the behaviour of materials upon irradiation, it appears that the description of the strain development process in irradiated materials is not precisely documented and understood. Furthermore, while numerous materials were already studied, they were all tackled separately and with different approaches; no work until now has dealt with several materials in a unique study using a consistent approach. Besides, it must be noted that the XRD analysis of irradiated materials at high fluence is not straightforward, owing in particular to the associated high disorder level that suppresses the specific features of XRD signals and prevents a reliable determination of the strain (see e.g. ${ }^{24,25,31}$ ). In the present paper, we specifically address all these issues by implementing an approach based on the use of a single experimental technique, namely XRD associated with a numerical simulation procedure ${ }^{46}$, combined with two complementary computational methods, namely molecular dynamics (MD) and rate equation cluster dynamics (RECD). We focus on four materials: uranium dioxide $\left(\mathrm{UO}_{2}\right)$, cubic zirconium dioxide $\left(\mathrm{c}-\mathrm{ZrO} \mathrm{H}_{2}\right)$, magnesium oxide $(\mathrm{MgO})$ and zirconium carbide $(\mathrm{ZrC})$; these materials exhibit various crystalline structures, bonding characters and defect properties (see Supplemental Material ${ }^{47}$ ). Monocrystalline samples have been irradiated in the 80 to $573 \mathrm{~K}$ temperature range to demonstrate the generality of the obtained results, but in the same energy-loss regime. Owing to the ion-energy range used (500 keV to $4 \mathrm{MeV}$ ), in the four cases, the energy deposition to the target-atom electrons, although it can be of the same magnitude as the energy transferred to the target (screened) nuclei, may be considered as having a weak influence on the defect generation process. Therefore, defects are predominantly produced during elastic collisions with the target atoms, and an estimation of the so-called ballistic damage is provided by the displacement per atom (dpa) parameter. The dpa parameter is directly related to the elastic energy-loss and to the number of ions impinging on the material (the fluence) and allows comparing a characterization parameter (such as the strain, as illustrated in the present work) determined under different irradiation conditions. It has been 
calculated with a dedicated code named SRIM (Stopping and Range of lons in Matter) ${ }^{48}$ that allows simulating ion/solid interactions (in amorphous structures) using a universal interaction potential.

We hereafter investigate the irradiation-induced strain development process in these four systems (i.e. different materials and irradiation conditions). Some differences are observed between experimental and computational results (these differences are addressed in the Supplemental Material $\left.{ }^{47}\right)$. Nevertheless, we shed light on the complete strain variation process by connecting the strain state to atomic defect structures, and furthermore, we unambiguously demonstrate that a wide range of materials exhibit the same characteristic behaviour, irrespective of the irradiation conditions and material properties. This statement is illustrated in Fig. 1 that displays the variation with dpa of the strain leve $\left.\right|^{49,50}$ as determined from XRD measurements. It clearly appears that in all cases, a two-step process is observed, with first a strain build-up followed by a strain relaxation (the difference in the strain magnitude and dpa level at which the relaxation takes place is related to the intrinsic characteristics of the materials, such as, e.g., the point defect mobility and the type of bonding). This strain development process is investigated in detail hereafter.

\section{Strain development process at the atomic scale: a combined MD and XRD investigation}

In order to catch the atomic details of the strain development in irradiated materials, we first focus on $\mathrm{UO}_{2}$. In this compound, it has been shown by $\mathrm{MD}$ simulations of collision cascades ${ }^{51}$ that the primary defects formed during ion irradiation are point defects ${ }^{52}$. Therefore, in order to mimic the ballistic damage without having to compute overlap of collision cascades, the Frenkel Pair Accumulation (FPA) technique was used, since it has already been proven very efficient to emulate irradiation damage in several materials ${ }^{27}{ }^{53-56}$. At different steps of the FPA process, XRD patterns were generated from the MD cells using the Debyer program ${ }^{57}$, as already implemented in $\mathrm{SiC}^{27}$ and pyrochlores ${ }^{55}$. The black curve in Fig. 2 represents the corresponding strain variation as a function of the displacement per uranium atom (dpU), which is equivalent to dpa except that oxygen atoms are disregarded in the estimation of this parameter, because only cation Frenkel pairs (FPs) were created 
(more details can be found $\mathrm{in}^{56}$ ). Note that the observed oscillations are simply due to bursts of FPs that have not yet been relaxed. We first observe an overall increase in the strain, and at a threshold $\mathrm{dpU}$ level, strain relaxation takes place. The analysis, using the DXA modifier of the Ovito $\operatorname{code}^{58}$, of the defects present in the MD cells at different irradiation levels reveals that their structure and density dramatically evolve during the whole irradiation sequence. It is not in the scope of this paper to provide a detailed mechanism of these defect changes, but it must be mentioned that such a mechanism, which involves the transformation of partial into perfect dislocation loops, has been described for fcc materials ${ }^{59}$. More importantly, the defect evolution is at the origin of the strain variation, as qualitatively described hereafter and quantitatively confirmed in the next section. At the beginning of the process, obviously, FPs are generated and these point defects are thus clearly at the origin of the strain build-up. Rapidly, different types of interstitial dislocation loops (shown in Fig. 3a) are formed due to interstitial defect clustering; these defects still strongly contribute to lattice strain. With increasing the $\mathrm{dpU}$ level, the dislocation loop size and density increase, as illustrated in Fig. 3b. Loop interactions eventually lead to the formation of a network of tangled dislocation lines, and to a considerable reduction in the loop density (Fig. 3c). This last defect transformation of dislocation loops into dislocation lines is at the origin of the release of the elastic strain, since the dislocation lines induce only a very weak elastic strain (but they generate heterogeneous strains that are usually referred to as microstrains); the effect of the different types of defects on the lattice parameter change is discussed in the next section. Our MD simulations therefore provide an explanation for both the strain build-up and strain relaxation processes in irradiated $\mathrm{UO}_{2}$. In Fig. 2, we have also plotted the experimental XRD data from $\mathrm{UO}_{2}$ single-crystals irradiated with $20 \mathrm{keV} \mathrm{He}{ }^{60}$ and $500 \mathrm{keV}$ Ce at room-temperature (RT). For both cases, the expected two-step strain development process is found (as partly shown in Fig. 1), and it is precisely what we observed with the MD-FPA technique. In addition, both the strain level and the $\mathrm{dpU}$ value at which the strain relaxation takes place exhibit a good agreement between experimental and computational data (a complete comparison is given in the Supplemental Materia $\left.\left.\right|^{47}\right)$. It is worth mentioning that the MD calculations were performed to be 
as general as possible and were not specifically designed to reproduce these two sets of experiments. Therefore, these results show the validity and the relevance of the MD calculations and of the corresponding data analysis.

The elastic-strain experimental data were derived from the simulation of the XRD curves (see Supplemental Material ${ }^{47}$ ) of irradiated $\mathrm{UO}_{2}$ crystals using a dedicated computer code ${ }^{46}$ that allows obtaining accurate strain depth-profiles. The $500 \mathrm{keV}$ Ce irradiation is presented as an illustrative case (Fig. 4a). To simulate the curves at high irradiation fluence, input from the MD calculations was needed: the strain was thus allowed to decrease and to potentially reach zero in the initial region of maximum damage as a consequence of dislocation line formation (at the expense of dislocation loops). In Fig.4, at low dpa level, the maximum strain is located close to the surface, and the strain plotted in Fig. 2 (and also in Fig. 1) is associated with this region. At high dpa, the strain is indeed relieved in this region. This strain relaxation observed in the strain profiles has not been shown before due to the lack of tangible evidences of such a process to occur, which is now filled by the present MD-FPA results that provide a clear picture of the complete strain behaviour and catch the detailed atomic scenario of the associated defect-structure changes.

Under ion irradiation, cubic $\mathrm{ZrO}_{2}$ and $\mathrm{MgO}$ exhibit a very similar overall behaviour as $\mathrm{UO}_{2}{ }^{36,61-63}$. It has been proposed, using TEM, that irradiation defects follow the same sequence of transformation in $\mathrm{c}-\mathrm{ZrO}_{2}{ }^{64}$ as that occurring in $\mathrm{UO}_{2}$ (and molecular dynamics simulations showed an identical process in cerium dioxide $\left.{ }^{65}\right)$. A comparable defect transformation sequence was observed in $\mathrm{MgO}^{62}$. Therefore, in the light of the strain development mechanism derived from the MD-FPA calculations in $\mathrm{UO}_{2}$, we provide a new interpretation of our experimental XRD data on $\mathrm{c}-\mathrm{ZrO}_{2}$ (irradiated with $4 \mathrm{MeV} \mathrm{Au}$ ) and $\mathrm{MgO}$ (irradiated with $1.2 \mathrm{MeV} \mathrm{Au}$ ). We deliberately chose two different irradiation temperatures, low (i.e. $80 \mathrm{~K}$ ) and intermediate (i.e. $573 \mathrm{~K}$ ) for $\mathrm{c}^{-} \mathrm{ZrO}_{2}{ }^{36}$ and $\mathrm{MgO}^{62,63}$, respectively, in order to show the common character of the proposed scenario of strain variation. Note however that, at RT, XRD curves in both materials exhibit a similar evolution with ion fluence as the one shown in the present work for the two selected temperatures ${ }^{36,62}$. Similarly to the 
$\mathrm{UO}_{2}$ case, in the region of maximum damage we allowed the strain to decrease due to the disappearance of dislocation loops. The strain depth-profiles are presented in Figs. 4b and 4c for $\mathrm{c}-\mathrm{ZrO}_{2}$ and $\mathrm{MgO}$, respectively. It is observed that the strain increases in the surface region at the beginning of the irradiation process. Then, the strain is relaxed at the initial strain peak with increasing dpa. Meanwhile, the maximum strain is shifted towards greater depth after this relaxation. These results are in agreement with those found in $\mathrm{UO}_{2}$. Consequently, the processes of strain build-up and strain relaxation presented for $\mathrm{UO}_{2}$ hold for $\mathrm{c}-\mathrm{ZrO}_{2}$ (and most likely for all materials with the fluorite structure such as the binary transuranium dioxides) but also for MgO which has a different crystalline structure and ionic character.

\section{Strain depth profiles and strain relaxation: a combined XRD and RECD approach}

In order to determine the strain depth profiles (due to the non-constant energy-loss deposition) with the twofold aim of supporting the proposed mechanism for the strain build-up and relaxation processes and demonstrating that these latter can be generalized to a great variety of materials, we investigated $\mathrm{ZrC}$ (see Supplemental Material ${ }^{47}$ for a comparison of the four materials characteristics). First, the XRD measurements were carried out on $\mathrm{ZrC}$ single-crystals irradiated with 1.2 MeV Au at RT at different ion fluences. The corresponding strain depth-profiles are plotted in Fig. 5a (experimental XRD data and associated simulations are presented in Supplemental Material $\left.{ }^{47}\right)$. Results are analogous to those observed for the previously studied materials. In addition to the XRD experiments, we implemented RECD simulations (performed here in a mean field approach with the 1D version of the CRESCENDO code ${ }^{66,67}$ ) to obtain, first, quantitative depth-profiles of the defect populations (which is not possible with the MD-FPA technique). Then, we developed a methodology to derive the strain depth-profiles from the RECD calculations (see ${ }^{68}$ for details in $\mathrm{ZrC}$ ) using the theory of linear elasticity, in order to compare them with those obtained experimentally. It has been shown that in $\mathrm{ZrC}$, vacancies are immobile at $\mathrm{RT}$, and they remain as mono-vacancies. On the contrary, interstitials are highly mobile and immediately tend to cluster to form dislocation 
loops $^{69,70}$. In the framework of linear elasticity, the strain induced by isolated vacancy point defects is proportional to their density $\left(N_{v}\right)$ and to their relaxation volume $\left(V_{V}^{\text {rel }}\right)^{70}$, which is -0.1 atomic volume $(\Omega)$ in $\mathrm{ZrC}$ as determined by density functional theory ${ }^{68}$; therefore, vacancies induce a weak lattice contraction. For isotropically distributed interstitial-type dislocation loops, the strain is proportional to the self-interstitial density inside the loops $\left(N_{l}\right)$, each interstitial increasing the lattice by one $\Omega^{70}$, which leads to a significant lattice expansion (that is however less than that induced by $N_{l}$ single interstitials having each a relaxation volume of a few $\left.\Omega^{70}\right)$. Finally, the total strain, $\varepsilon$, reads as:

$$
\varepsilon=N_{V} V_{V}^{r e l}+N_{I} \Omega
$$

Subsequently, in order to reproduce the strain relaxation process, we introduced a mechanism of dislocation loop interaction that leads to the formation of entangled dislocation lines; note that we also introduced a reasonable density of intrinsic dislocation lines, i.e. $10^{9} \mathrm{~cm}^{-3}$. This mechanism $\mathrm{f}$ dislocation line formation begins when dislocation loops start to come into contact, i.e. when their size distribution and density allow these interactions. At that point, the density of the dislocation lines increases, at the expense of loops, thereby strongly decreasing the strain rate and eventually leading to strain relaxation because the lattice parameter change induced by a dislocation line reduces to the dislocation core volume that is approximately that of a row of self-interstitials (hence much less than $N_{l} \Omega$ ). This phenomenon is marginal for small dislocation loops but becomes significant for loops of radius $\sim 1 / \sqrt{\pi \rho_{t}}$, where $\rho_{t}$ is the total dislocation density accounting for loops and network dislocations ${ }^{71}$. Furthermore, as the size of the dislocation lines reaches the primary extinction length of X-rays (i.e. in the micrometer range), $\mathrm{X}$-ray coherent scattering can no longer correlate atomic positions and elastic strain cannot be measured anymore (XRD peak shift is replaced by peak broadening). Therefore, for the RECD calculations, the volume change induced by the dislocation lines was set to zero. This approach mimics the mechanism evidenced by our MD simulations in $\mathrm{UO}_{2}$ and further confirmed in $\mathrm{c}^{-\mathrm{ZrO}_{2}}$ and $\mathrm{MgO}$, and it allows a comparison with the XRD experiments. 
Using Eq.(1) and the distributions of the defect populations, we computed the elastic-strain depth profiles at different dpa levels in ZrC (see Fig. 5b). At low dpa, the strain is maximum close to the surface and it increases with irradiation fluence until it starts to decrease, leading to an apparent peak shift to greater depth. This result indicates that the strain is primarily relaxed at the initial strain peak, i.e. where the interstitial production rate is the highest, thus ensuring a faster clustering and transformation into dislocation lines. With increasing the dpa level, the strain relaxation is more pronounced; meanwhile, the apparent maximum strain, which keeps on shifting to greater depth, also starts diminishing. These results match the findings obtained experimentally (Fig. 4a). There exists a difference in the depth scales that is discussed in the Supplemental Material ${ }^{47}$. On the contrary, notably, the strain levels are very close to those determined by XRD. Similarly, the dpa level at which the strain relaxation takes place is on the same order of magnitude as the one determined experimentally. Therefore, a good overall agreement between experiments and RECD simulations demonstrates that the proposed mechanism for the strain build-up and subsequent relaxation processes also holds for $\operatorname{ZrC}$ (and our methodology to compute the strain depth-profiles with the RECD technique is validated).

\section{Conclusion}

We first exemplified through experimental XRD data (see Fig. 1) the prevalent development process of strain in irradiated materials that is defined by a strain build-up followed by a strain relaxation. This prevalent process is then rationalized thanks to modelling by two computational techniques, namely molecular dynamics Frenkel pair accumulation and rate equation cluster dynamics. These calculations allowed proposing a general mechanism for this two-step process. Owing to their large clustering tendency, irradiation-induced interstitial point defects rapidly form extended defects (mainly dislocation loops). These defects, that induce a significant elastic strain (i.e. a lattice volume change), are progressively incorporated into a network of tangled dislocation lines, thereby inducing a substantial decrease in the strain rate and thus a strain relaxation. These results 
provide a description that reconciles previous studies on this topic. Moreover, the strain depth profiles associated to the non-constant energy deposition of ions are quantitatively determined by experiments and computations and both sets of data show a reasonable agreement. To finish, we provide a methodology combining experimental and computational work that can be used by the researchers involved in the field of ion-beam-induced effects in materials. 


\section{Acknowledgments}

$A D, S P, D B-P, T-H N, L T$ and FG are grateful to the SEMIRAMIS staff for their help during ion irradiation experiments. Authors would like to warmly thank E. Clouet from CEA-SRMP for fruitful discussions on the linear elasticity of defects. AD, DB-P and LT sincerely thank M. Béhar from Instituto de Fisica of the Universidade Federal of Rio Grande do Sul for the $80 \mathrm{~K}$ irradiations of $\mathrm{c}-\mathrm{ZrO}_{2}$. $\mathrm{AD}, \mathrm{AB}, \mathrm{T}-\mathrm{HN}$ and FG acknowledge V. Demange from Institut des Sciences Chimiques de Rennes for her help during the XRD measurements on $\mathrm{UO}_{2}$. The nanocenter CTU-IEF-Minerve, where part of the XRD measurements has been performed, is partially funded by the 'Conseil Général de l'Essonne'. The work on c-ZrO and $\mathrm{MgO}$ has been partially supported by the NEEDS-Matériaux program of the CNRS. DB-P acknowledges Campus France for the Eiffel Excellence scholarship and Universidad Autónoma de Madrid for the FPI scholarship. JC is thankful to Région Limousin for providing a PhD grant. 


\section{Figures}

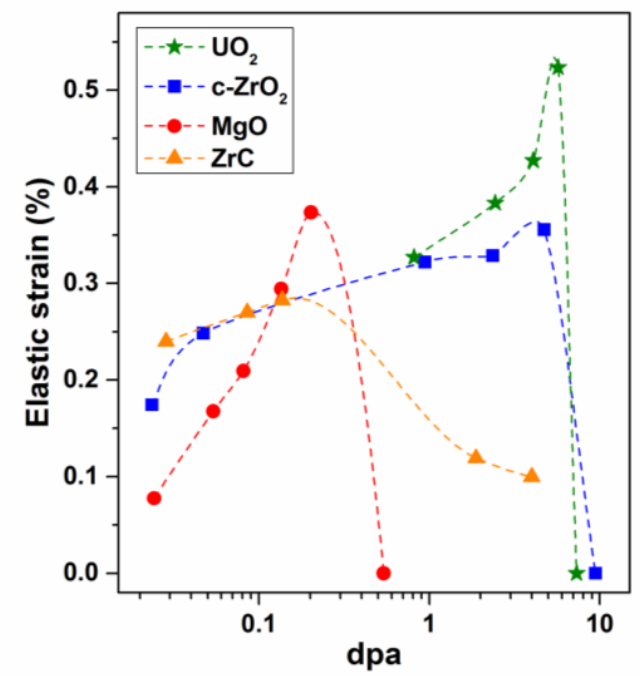

Figure 1: Elastic strain level as determined from XRD data obtained for four different systems: (i) $\mathrm{UO}_{2}$ irradiated with $500 \mathrm{keV} \mathrm{Ce}$ at RT (stars), (ii) c- $\mathrm{ZrO}_{2}$ irradiated with $4 \mathrm{MeV} \mathrm{Au}$ at $80 \mathrm{~K}$ (squares), (iii) MgO irradiated with $1.2 \mathrm{MeV}$ Au at $573 \mathrm{~K}$ (circles) and (iv) ZrC irradiated with $1.2 \mathrm{MeV} \mathrm{Au}$ at RT (triangles). Data were taken at the initial strain peak (see Figures 3 and 4 in the main article and the corresponding XRD curves presented in Fig.1 of the Supplemental Material ${ }^{47}$ ). Lines are drawn for visualization purposes. 


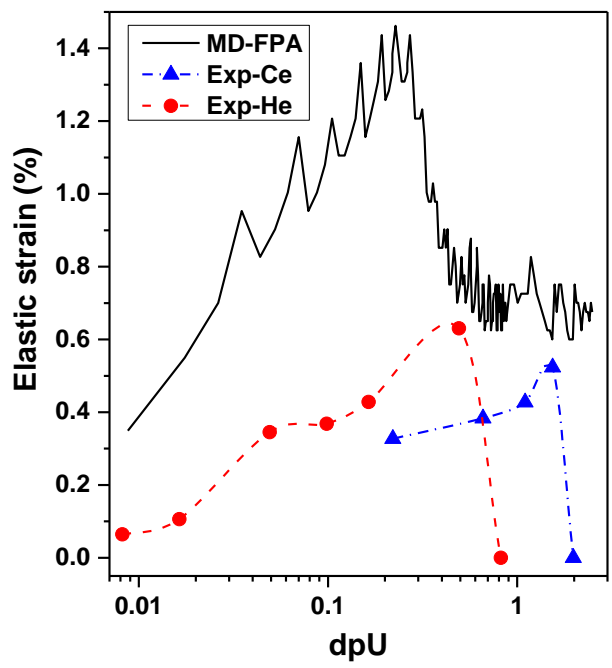

Figure 2: Variation with $\mathrm{dpU}$ of the elastic strain level in $\mathrm{UO}_{2}$ as determined from $\mathrm{XRD}$ patterns obtained by MD-FPA simulations (black line) and experiments (circles for He irradiation and triangles for Ce irradiation); dashed and dash-dotted lines are drawn for visualization purposes. 


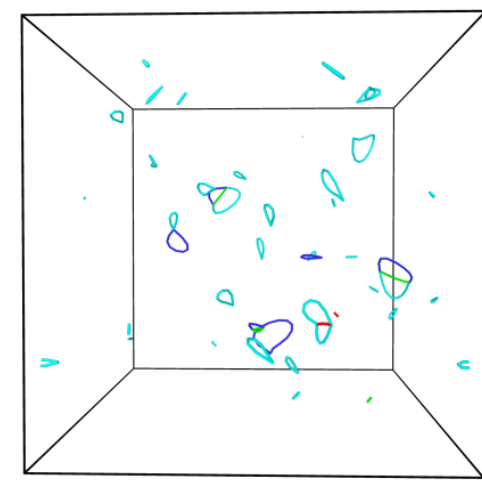

a

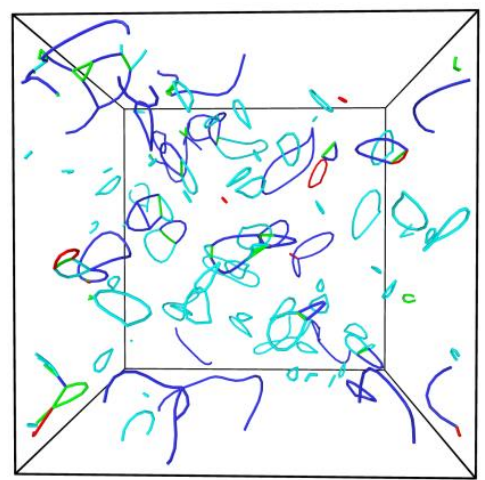

b

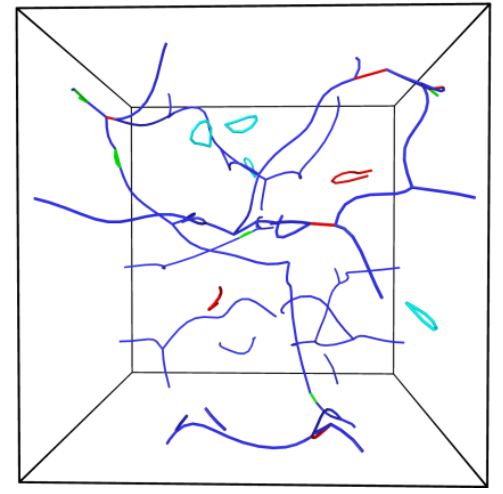

C

Figure 3: Snapshots obtained by using the DXA modifier of the OVITO code ${ }^{58}$ with the molecular dynamics simulations of Frenkel pair accumulation (FPA) in $\mathrm{UO}_{2}$, and showing the change of the defect nature with ion fluence: a) at $0.12 \mathrm{dpU}$, in addition to FP (not shown here), various types - as materialized by different colors - of dislocations loops are formed; b) at $0.21 \mathrm{dpU}$, strain is at its maximum (see Fig. 2), and the snapshot shows a high density of dislocation loops that are on the verge of transforming into dislocation lines; $c$ ) at $0.95 \mathrm{dpU}$, the transformation of loops into lines is almost complete, and a network of dislocation lines has developed. 
a) UO - 500 keV Ce @ RT

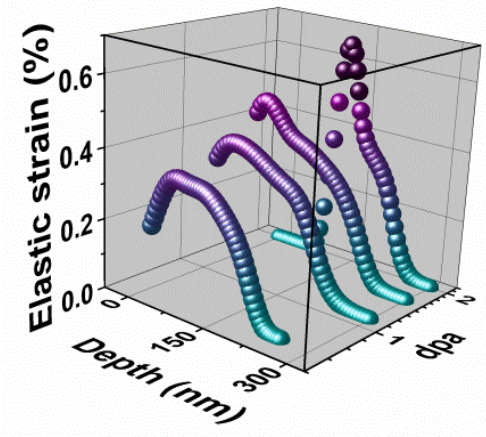

b) $\mathrm{c}-\mathrm{ZrO}_{2}-4 \mathrm{MeV} \mathrm{Au} @ 80 \mathrm{~K}$

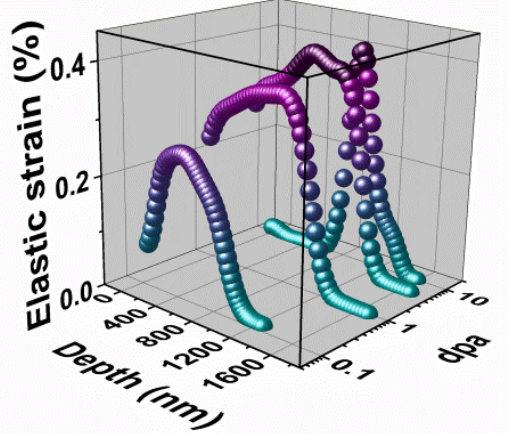

c) MgO - 1.2 MeV Au @ 573 K

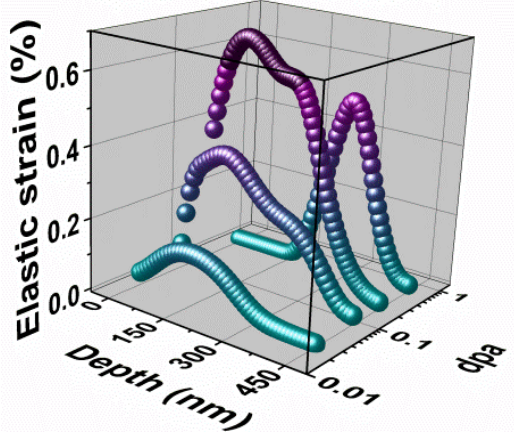

Figure 4: Elastic-strain depth profiles obtained from the fitting of experimental XRD data recorded on irradiated a) $\mathrm{UO}_{2}$, b) $\mathrm{c}-\mathrm{ZrO}_{2}$ and c) $\mathrm{MgO}$ single-crystals; the third axis (on the right side of the figures) indicates the dpa level. The XRD curves along with the corresponding fits are presented in Fig.1 of the Supplemental Material ${ }^{47}$. 
a) ZrC - 1.2 MeV Au @ RT - XRD

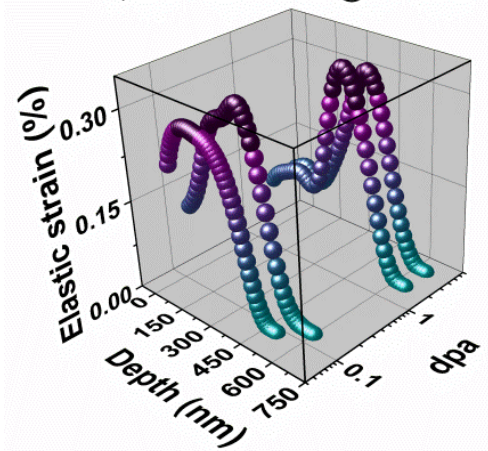

b) ZrC - 1.2 MeV Au @ RT - RECD

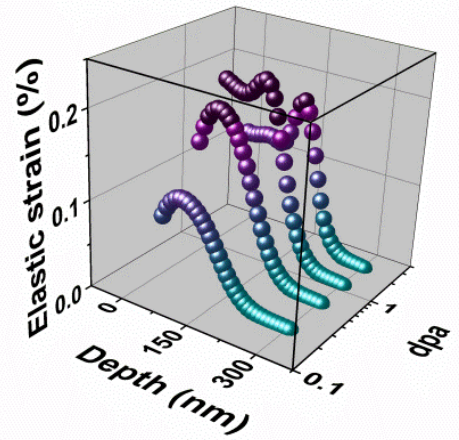

Figure 5: Elastic-strain depth profiles in irradiated ZrC irradiated obtained from a) XRD experimental data and b) RECD simulations (note that data were interpolated and the number of slices was doubled in order to smoothen the curves); the third axis (on the right side of the figures) indicates the dpa level. The XRD curves along with the corresponding fits are presented in Fig.1 of the Supplemental Material ${ }^{47}$. 


\section{References}

1. M. Nastasi, J. W. Mayer, Ion Implantation and Synthesis of Materials, Springer Eds (2006)

2. T. Som, D. Kanjilal, Nanofabrication by lon-Beam Sputtering: Fundamentals and Applications, CRC Press, Taylor and Francis Group (2012)

3. N. Yao, Focused Ion Beam Systems, MaterialsToday 10, 53 (2007)

4. M. Lang, F. Zhang, J. Zhang, J. Wang, B. Schuster, C. Trautmann, R. Neumann, U. Becker, R.C. Ewing, Nanoscale manipulation of the properties of solids at high pressure with relativistic heavy ions, Nature Materials 8, 793 (2009)

5. O. Ochedowski, O. Osmani, M. Schade, B. K. Bussmann, B. Ban-d'Etat, H. Lebius, M. Schleberger, Graphitic nanostripes in silicon carbide surfaces created by swift heavy ion irradiation, Nature Communications 5, 3913 (2014)

6. K. Nordlund, J. Keinonen, M. Ghaly, R. S. Averback, Coherent displacement of atoms during ion irradiation, Nature 398, 49 (1999)

7. B. P. Uberuaga, M. Tang, C. Jiang, J. A. Valdez, R. Smith, Y. Wang, K. E. Sickafus, Opposite correlations between cation disordering and amorphization resistance in spinels versus pyrochlores, Nature Communications 6, 8750 (2015)

8. Y. Zhang, A. Debelle, A. Boulle, P. Kluth, F. Tuomisto, Advanced Techniques for Characterization of Ion Beam Modified Materials, Current Opinion in Solid State \& Materials Science 19, 19 (2015)

9. H. C. Huang, J. I. Dadap, G. Malladi, I. Kymissis, H. Bakhru, R. M. Jr Osgood, Helium-ion-induced radiation damage in $\mathrm{LiNbO}_{3}$ thin-film electro-optic modulators, Opt. Express 22, 19653 (2014)

10. T. Ozaki, L. Wu, C. Zhang, J. Jaroszynski, W. Si, J. Zhou, Y. Zhu, Q. Li, A route for a strong increase of critical current in nanostrained iron-based superconductors, Nature Communications 7, 13036 (2016)

11. N. Theodoropoulou, A. F. Hebard, M. E. Overberg, C. R. Abernathy, S. J. Pearton, S. N. G. Chu, R. G. Wilson, Unconventional Carrier-Mediated Ferromagnetism above Room Temperature in lonImplanted (Ga, Mn)P:C, Phys. Rev. Lett. 89, 107203 (2002)

12. A. Redondo-Cubero, K. Lorenz, S. Magalhães, E. Alves et al., Analysis of the stability of InGaN/GaN multiquantum wells against ion beam intermixing", Nanotechnology 26, 425703 (2015) 
13. Y. Zhang, M. Ishimaru, T. Varga, T. Oda, C. Hardiman, H. Xue, et al., Nanoscale engineering of radiation tolerant silicon carbide, Phys Chem Chem Phys. 14, 13429 (2012)

14. D. V. Potapenko, Z. Li, J. W. Kysar, R. M. Osgood, Nanoscale strain engineering on the surface of a bulk $\mathrm{TiO}_{2}$ crystal, Nanoletters 14, 6185 (2014)

15. R. Gupta, N. Sehdev, K. Asokan, D. Kanjilal, S. Annapoorni, Engineering strain, densification, order parameter and magnetic properties of FePt thin films by dense electronic excitations, J. Appl. Phys. $116,083902(2014)$

16. H. Guo, S. Dong, Ph. D. Rack, J. D. Budai, Ch. Beekman, Z. Gai, W. Siemons, C. M. Gonzalez, R. Timilsina, A. T. Wong, A. Herklotz, P. C. Snijders, E. Dagotto, Th. Z. Ward, Strain Doping: Reversible Single-Axis Control of a Complex Oxide Lattice via Helium Implantation, Phys. Rev. Lett 114, 256801 (2015)

17. Y. H. Li, B. P. Uberuaga, C. Jiang, S. Choudhury, J. A. Valdez, M. K. Patel, J. Won, Y.-Q. Wang, M. Tang, D. J. Safarik, D. D. Byler, K. J. McClellan, I. O. Usov, T. Hartmann, G. Baldinozzi, K. E. Sickafus, Role of Antisite Disorder on Preamorphization Swelling in Titanate Pyrochlores, Phys. Rev. Lett 108, 195504 (2012)

18. G. S. Was, Z. Jiao, E. Getto, K. Sun, A. M. Monterrosaa, S. A. Maloy, O. Anderoglu, B. H. Sencer, M. Hackett, Emulation of reactor irradiation damage using ion beams, Scripta Mater. 88, 33 (2014)

19. N. M. Anoop Krishnan, B. Wang, Y. Yu, Y. Le Pape, G. Sant, M. Bauchy, Phys. Rev. X 7, 031019 (2017)

20. A. Mozumder, Y. Hatano, Charged Particle and Photon Interactions with Matter: Chemical, Physicochemical, and Biological Consequences with Applications (chapter 4), Marcel Dekker Inc Eds, New York (2004)

21. S. J. Pearton, F. Ren, E. Patrick, M. E. Law, A. Y. Polyakov, lonizing Radiation Damage Effects on GaN Devices, ECS Journal of Solid State Science and Technology 5, Q35 (2016)

22. M. Nastasi, J. W. Mayer, J. K. Hirvonen, Ion-solid interactions: fundamentals and applications, Cambridge University Press Eds, Cambridge (1996)

23. S. Rao, B. He, C. R. Houska, X-ray diffraction analysis of concentration and residual stress gradients in nitrogen-implanted niobium and molybdenum, J Appl Phys 69, 8111 (1991) 
24. S. Decoster, A. Vantomme, Implantation-induced damage in Ge: strain and disorder profiles during defect accumulation and recovery, J. of Phys. D: Appl.Phys. 42, 165404 (2009)

25. D. Bachiller-Perea, A. Debelle, L. Thomé, J.-P. Crocombette, Study of the initial stages of defect generation in ion-irradiated $\mathrm{MgO}$ at elevated temperatures using high-resolution $\mathrm{X}$-ray diffraction, J. Mater. Sci. 51, 1456 (2016)

26. A. Boulle, A. Debelle, Statistical nature of atomic disorder in irradiated materials, Phys. Rev. Lett. $116,245501(2016)$

27. A. Debelle, A. Boulle, A. Chartier, F. Gao, W. J. Weber, Interplay between atomic disorder, lattice swelling and defect energy in ion irradiation induced amorphization of SiC, Phys. Rev. B. 90, 174112 (2014)

28. V. S. Speriosu, B. M. Paine, M.-A. Nicolet, L. H. Glass, X-ray rocking curve study of Si-implanted GaAs, Si, and Ge, Appl. Phys. Lett. 40, 604 (1982)

29. M. Servidori, Characterization of lattice damage in ion implanted silicon by multiple crystal $x$-ray diffraction, Nucl. Instr. Meth. B 19-20, 443 (1987)

30. P. Partyka, R. S. Averback, D. V. Forbes, J. J. Coleman, X-ray diffraction and channeling-Rutherford backscattering spectrometry studies of ion implantation damage in $\mathrm{Al}_{x} \mathrm{Ga}-\mathrm{x} A \mathrm{As}, \mathrm{J}$. Appl. Phys. 83, 1265 (1998)

31. B. Lacroix, S. Leclerc, A. Declémy, K. Lorenz, E. Alves, P. Ruterana, Mechanisms of damage formation in Eu-implanted GaN probed by X-ray diffraction, Eur. Phys. Lett. 96, 46002 (2011)

32. A. Turos, On the mechanism of damage buildup in gallium nitride, Rad. Eff. Def. Solids 168, 431 (2013)

33. N. Nakae, A. Harada, T. Kirihara, S. Nasu, Irradiation induced lattice defects in $\mathrm{UO}_{2}$, J. Nucl. Mater. 71, 314 (1978)

34. J. Spino, D. Papaioannou, Lattice parameter changes associated with the rim-structure formation in high burn-up $\mathrm{UO}_{2}$ fuels by micro X-ray diffraction, J. Nucl. Mater. 281, 146 (2000)

35. T. Yang, C. A. Taylor, Sh. Kong, Ch. Wang, Y. Zhang, X. Huang, J. Xue, Sha Yana, Y. Wang, The discrepancies in multistep damage evolution of yttria-stabilized zirconia irradiated with different ions, J. Nucl. Mater. 443, 40 (2013) 
36. A. Debelle, J. Channagiri, L. Thomé, B. Décamps, A. Boulle, S. Moll, F. Garrido, M. Behar, J. Jagielski, Comprehensive study of the effect of the irradiation temperature on the behavior of cubic zirconia, J. Appl. Phys 115, 183504 (2014)

37. K. Papamihail, K. Mergia, F. Ott, Y. Serruys, Th. Speliotis, G. Apostolopoulos, S. Messoloras, $\mathrm{Fe}^{+}$ion irradiation induced changes in structural and magnetic properties of iron films, Nucl. Mater. Energy 9, 459 (2016)

38. D. Yun, Y. Miao, R. Xu, Z. Mei, K. Mo, W. Mohamed, B. Ye, M. J. Pellin, A. M. Yacout, Characterization of high energy Xe ion irradiation effects in single crystal molybdenum with depthresolved synchrotron microbeam diffraction, J. Nucl. Mater. 471, 272 (2016)

39. M. W. Ullah, Y. Zhang, N. Sellami, A. Debelle, W. J. Weber, H. Bei, W. J. Weber, Evolution of irradiation-induced strain in an equiatomic NiFe alloy, Scripta Mater, submitted

40. R. Kumar, W. Khan, J. P. Srivastava, S. K. Arora, R. G. S. Sofin, R. J. Choudhary, I. V. Shvets, Swift heavy ion irradiation-induced modifications in structural, magnetic and electrical transport properties of epitaxial magnetite thin films, J. Appl. Phys. 100, 033703 (2006)

41. G. Sattonnay, C. Grygiel, I. Monnet, C. Legros, M. Herbst-Ghysel, L. Thomé, Phenomenological model for the formation of heterogeneous tracks in pyrochlores irradiated with swift heavy ions, Acta Mater 60, 22 (2012)

42. C. A. Volkert, Stress and plastic flow in silicon during amorphization by ion bombardment, J. Appl. Phys. 70, 3521 (1991)

43. S. L. Ellingboe, M. C. Ridgway, The production and stability of implantation-induced vacancy excesses in silicon, Nucl. Instrum. Methods Phys. 127, 90 (1997)

44. L. Raghu, S. Hatt, P. Kluth, S. M. Kluth, R. Dogra, M. C. Ridgway, Variation of ion-irradiation induced strain as a function of ion fluence in Si, Nucl. Instrum. Methods Phys. Res. B 257, 236 (2007)

45. A. Boulle, A. Debelle, J. B. Wallace, L. B. Bayu Aji, S. O. Kucheyev, The amorphization of 3C-SiC irradiated at moderately elevated temperatures as revealed by X-ray diffraction', Acta Mater. 140, $250(2017)$

46. M. Souilah, A. Boulle, A. Debelle, RaDMaX: a graphical program for the determination of strain and damage profiles in irradiated crystals, J. Appl. Cryst. 49, 311 (2016)

47. Supplemental material, see 
48. J.F. Ziegler, J. P. Biersack, U. Littmark, The Stopping and Range of lons in Solids, Pergamon, New York, 1985. Available at: www.srim.org.

49. Note that in the present paper, the strain only due to irradiation defects is shown, and the mechanical contribution of the un-irradiated part of the materials has been removed, as explained in a few recent papers (see ${ }^{50}$ and references therein).

50. A. Debelle, A. Boulle, F. Rakotovao, J. Moeyaert, C. Bachelet, F. Garrido, L. Thomé, Influence of elastic properties on the strain induced by ion irradiation in crystalline materials, J. Phys. D: Appl. Phys. 46, 045309 (2013)

51. A collision cascade is a large number of successive elastic collisions involving numerous target atoms and initiated by a primary knock-on (target) atom after an incident ion transferred it enough energy to induce this collective displacement phenomenon

52. L. Van Brutzel, M. Rarivomanantso, D. Ghale, Displacement cascade initiated with the realistic energy of the recoil nucleus in $\mathrm{UO}_{2}$ matrix by molecular dynamics simulation, J. Nucl. Mater. 354, 28 (2006)

53. J.-P. Crocombette, A. Chartier, W. J. Weber, Atomistic simulation of amorphization thermokinetics in lanthanum pyrozirconate, Appl. Phys. Lett. 88, 051912 (2006)

54. A. Chartier, G. Catillon, and J.-P. Crocombette, Key role of the cation interstitial structure in the radiation resistance of pyrochlores, Phys. Rev. Lett. 102, 155503 (2009)

55. A. Soulié, D. Menut, J.-P. Crocombette, A. Chartier, N. Sellami, G. Sattonnay, I. Monnet, J.-L. Béchade, $\mathrm{X}$-ray diffraction study of the $\mathrm{Y}_{2} \mathrm{Ti}_{2} \mathrm{O}_{7}$ pyrochlore disordering sequence under irradiation, J. Nucl. Mater. 314, 480 (2016)

56. A. Chartier, C. Onofri, L. Van Brutzel, C. Sabathier, O. Dorosh, J. Jagielski, Early stages of irradiation induced dislocations in urania, Appl. Phys. Lett. 109, 181902 (2016)

57. Wojdyr, Debyer software, 2008. https://debyer.readthedocs.io/en/latest/

58. A. Stukowski, V.V. Bulatov, A. Arsenlis, Automated identification and indexing of dislocations in crystal interfaces, Modelling Simul. Mater. Sci. Eng. 20, 085007 (2012); www.ovito.org

59. D. Hull, D.J. Bacon, Introduction to dislocations, $5^{\text {th }}$ edition, Elsevier (2011)

60. A. Debelle, A. Boulle, F. Garrido, L. Thomé, Strain and stress build-up in He-implanted $\cup_{2}$ single crystals: an X-ray diffraction study, J. Mater. Sci. 46, 4683 (2011) 
61. T.-H. Nguyen, A. Debelle, A. Boulle, F. Garrido, L. Thomé, V. Demange, Mechanical response of $\mathrm{UO}_{2}$ single crystals submitted to low-energy ion irradiation, J. Nucl. Mater. 467, 505 (2015)

62. S. Moll, Y. Zhang, A. Debelle, L. Thomé, J.-P. Crocombette, Z. Zihua, J. Jagielski, W.J. Weber, Damage processes in MgO irradiated with medium-energy heavy ions', Acta Mater. 88, 314 (2015)

63. D. Bachiller-Perea, A. Debelle, L. Thomé, M. Behar, Damage accumulation in MgO irradiated with MeV Au ions at elevated temperatures', J. Nucl. Mater. 478, 268 (2016)

64. B. Baufeld, D. Baither, U. Messerschmidt, M. Bartsch, I. Merkel, In Situ Study on the Generation of Radiation Damage in Cubic-Zirconia in the High-Voltage Electron Microscope, J. Am. Ceram. Soc. 76, 3163 (1993)

65. Y. Miao, D. Aidhy, W.-Y Chen, K. Mo, A. Oaks, D. Wolf, J. F. Stubbins, The evolution mechanism of the dislocation loops in irradiated lanthanum doped cerium oxide, J. Nucl. Mater. 445, 209 (2014)

66. T. Jourdan, G. Bencteux, G. Adjanor, Efficient simulation of kinetics of radiation induced defects: A cluster dynamics approach, Journal of Nuclear Materials 444, 298 (2014)

67. T. Jourdan, G. Stoltz, F. Legoll, L. Monasse, An accurate scheme to solve cluster dynamics equations using a Fokker-Planck approach, Comp. Phys. Comm. 207, 170 (2016)

68. S. Pellegrino, J.-P. Crocombette, A. Debelle, Th. Jourdan, L. Thomé, Multi-scale simulation of the experimental response of ion-irradiated zirconium carbide: Role of interstitial clustering, Acta Mater. 102,79 (2016)

69. L. Van Brutzel, J.-P. Crocombette, Classical molecular dynamics study of primary damage created by collision cascade in a ZrC matrix, Nucl. Instr. Meth. B 255, 141 (2007)

70. P. H. Dederichs, C. Lehman, H. R. Schober, A. Scholz, R. Zeller, Lattice theory of point defects, J. Nucl. Mater. 69-70, 176 (1978)

71. T. Jourdan, Influence of dislocation and dislocation loop biases on microstructures simulated by rate equation cluster dynamics, J. Nucl. Mater. 467, 286 (2015) 